Virginia Dignum, Christiaan Tick: Agent-based Analysis of Organizations: Performance and Adaptation, IAT'07, November 2007 


\section{Agent-based Analysis of Organizations: Formalization and Simulation}

\author{
Virginia Dignum \\ Utrecht University, The Netherlands \\ email: virginia@cs.uu.nl
}

\author{
Christiaan Tick \\ Utrecht University, The Netherlands \\ christiaan.tick@phil.uu.nl
}

\begin{abstract}
Organizational effectiveness depends on many factors, including individual excellence, efficient structures, effective planning and capability to understand and match context requirements. We propose a way to model organizational performance based on a combination of formal models and agent-based simulation that supports the analysis of the congruence of different organizational structures to changing environments.
\end{abstract}

\section{Introduction}

Organizational adaptation is the process to evaluate the how an organization matches its environment, and to determine the changes leading to better performance given those environmental conditions. That is, it is assumed that organizations try to locate the structure that best fits their environment and will make changes in their design accordingly [1]. Providing decision-makers with knowledge and tools that enable them to effectively identify, assess and respond to environmental changes is of utmost importance for the survival of the organization. Our research aims at developing tools and formalisms to model organizations and evaluate their performance under different circumstances. On the one hand, we have developed a modelling language with formal semantics that enables the specification of abstract organization models, the verification of formal properties such as flexibility or robustness [4], and the analysis of organizational adaptation in dynamic environments. On the other hand, we developed a simulation framework to describe environment characteristics and the response of different organizational designs to those characteristics. Simulation results can be used to inform strategic decisions concerning reorganization [3].

Formal systems enable to represent organizations, their environment, objectives and agents such that their partial contributions to the performance of the organization in a changing environment can be understood. Moreover, such models must be realistic enough to incorporate the more pragmatic considerations faced by real organizations. Most existing formalisms lack this realism, either by ignoring temporal issues, by taking a very restrictive view on the controllability of agents, or by assuming complete control and knowledge within the systems.

Computational simulations are based on formal models, in the sense that they provide a precise theoretical formulation of relationships between variables. Simulations provide a powerful way to analyze and construct realistic models of organizational systems and make possible to study problems that are not easily addressed by other scientific approaches [5]. Such formal models are however limited to the specific domain and difficult to validate. Techniques are thus needed that make possible the formal validation, comparison and extendability of simulation models. The language presented in this paper, based on modal logic is a first attempt to provide such a meta model for reasoning about computational models, that has both a formal semantics as well as the capability to represent realistic concepts.

\section{Formal Model of Organization}

Organizations are complex dynamic goal-oriented processes, based on the coordinated and purposeful action of human beings in the environment. Three broad classes of characteristics jointly determine organizational performance[6]. The first are the structural factors, which are the components and features of the organization (such as roles, dependencies, constraints, norms and regulations). The second are task environmental factors, which are the components and features of the task (such as size, time constraints, uncertainty). The third class of factors are agent factors, which are the characteristics of the individual agents concerning task capability, intelligence (including decision making and reasoning capabilities), social awareness, etc. These three classes of factors jointly determine the performance of the organization. Formally, given a set of worlds $W$ representing states of affairs in the environment, an organization $O$ in a world $w \in W$ is defined as:

$O^{w}=\left\{A_{O}, \leq_{O}, D_{O}, S_{O}\right\}$ where $A_{O}^{w}=\left\{a_{1}, \ldots, a_{n}\right\}$ is the set of agents, $\left(A_{O}^{w}, \leq_{O}^{w}\right)$ is 
a partial order relation on $A$ reflecting the structure of the organization, $D_{O}^{w} \subseteq \Phi$ is a set of objectives (states of affairs to achieve), and $S_{O}^{w} \subseteq \Phi$ is the set of current assets and characteristics of $O$. Due to space limitations, we cannot describe here the full model, but refer the reader to [2].

Agents can control the state of variables in a world, represented by $C_{a} \varphi$, and furthermore are able to act on the world and as such bring states of affairs to happen, represented by $E_{a} \varphi$, meaning that agent $a$ sees to it that fact $\varphi$ holds in all next worlds. However, agents are limited on their capabilities, that is, the set of facts in the world that they can control. This implies that in MAS certain states of affairs can only be reached if two or more agents cooperate to bring that state to be. We define control and action of a group of agents based on the combined atomic capabilities of the agent in the group. For example, if a group contains two agents $a$ and $b$, such that $C_{a}$ sand-door and $C_{b}$ paintdoor, only working as a group is possible to realize both. This is represented by $C_{\{a, b\}}$ (sand-door and paint-door).

The objectives of an organization, $D_{O}$, are achieved through agent action. In order to make this possible, an organization must employ the relevant agents. Furthermore, one of the main reasons for creating organizations is efficiency, that is, to provide the means for coordination that enable the achievement of global goals in an efficient manner. Dependency between agents $a$ and $b$ is represented by $a \leq b$ meaning that agent $a$ is able to delegate the realization of some state of affairs to agent $b$. Agents can be made responsible for some of the objectives of the organization, represented by $R_{a} \varphi$. Responsibility means that either the agent attempts to achieve that objective, or it delegates the objective to another agent with whom it has a dependency relation. That is, given an organization $O=\left(\{a, b\}, a \leq_{O} b, D_{O}, S_{O}^{w}\right)$ and a proposition $\varphi$, such that $R_{a} \varphi$, the order relation $a \leq_{O} b$ indicates that $a$ is capable to pass its responsibility for $\varphi$ to $b$, that is, $C_{a} R_{b} \varphi$. We refer to [2] for the formal specification.

This abstract definition of organizations enables to analyze some theoretical properties and incorporates some realistic notions such as bounded capabilities of agents, coordination and control. As an example, consider the organization $O=\left(\left(A, \leq_{O}\right), D, S^{0}\right)$, where:

$A$ is the set of agents $\{a, b, c, d\}$,

$\leq_{O}$ are dependencies between the agents:

$\left.\left(a \leq_{O} b, a \leq_{O} c \leq_{O} d\right)\right)$,

$D$ is the objective of the organization:

$D=($ sand-door $\wedge$ paint-door $) \vee$ tile-floor $\vee$ paper-wall

$S^{0}$ is the initial state:

$\left\{R_{a} D, C_{b}\right.$ sand-door, $C_{d}$ paint-door, $C_{\{b, c\}}$ tile-floor $\}$

Note that the initial organizational state $S^{0}$ indicates the capabilities of the agents in $A$ and that agent $a$ is responsible for the achievement of the organizational goal. This example also shows that organizations are dependent on the ca- pabilities of their agents to achieve their objectives. In this case, without agent $b$, the organization can never achieve its goals. A possible strategy for this organization to realize the organizational objectives is:

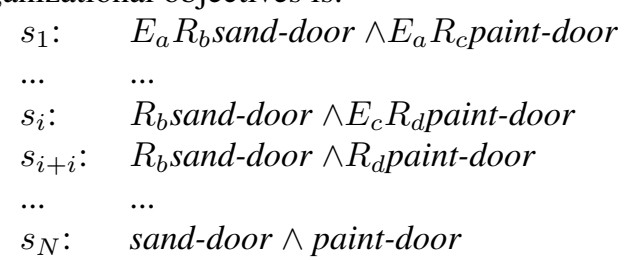

\section{Adapting organization design}

Changes in the environment lead to alterations on the effectiveness of the organization and therefore in a need to reorganize, or at least, the need to consider the consequences of the change to the organization's effectiveness and possibly efficiency. In our model, a successful organization, is such that $D_{O} \subseteq S_{O}$, Reorganization activities aim therefore at aligning these sets, either by attempting to change the current state, or by altering the set of desires. Basically, reorganization consists of two activities: the evaluation of current organizational state and its 'distance' to desired state, and, the choice of reorganization strategy, that is, the purposeful change of organizational constituents (structure, agent population, objectives) in order to make a path to desired state possible and efficient. Reorganization activities can be classified in three groups:

- Staffing: Changes on the set of agents: adding new agents, or deleting agents from the set. Corresponding to personnel activities in human organizations (hiring, firing and training). Staffing operators are $\operatorname{staff}^{+}(O, a)$ and $\operatorname{staff}^{-}(O, a)$.

- Structuring Changes on the ordering structure of the organization. Corresponding to infrastructural changes in human organizations: e.g. changes in composition of departments or positions. Structuring operators are

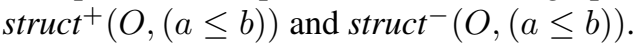

- Strategy Changes on the objectives of the organization: adding or deleting desired states. Corresponding to strategic (or second-order) changes in human organizations: on the mission, vision, or charter of the organization. Strategy operators are strateg $^{+}(O, d)$ and strateg $^{-}(O, d)$.

The classification above is very generic, but it allows for the representation of all different types of modifications that can be performed on the formal definition of organization we use. Furthermore, most realistic adaptation possibilities can be represented in this classification. For instance, a change of role allocation, that is changing the capabilities of an agent enacting a role, is represented by the deletion of 
one agent and the addition of a new one, with the new capabilities. The organizational model represents organizational strategy as objectives, abstracting from e.g. the notion of plan. These objectives represent desired states of the world (which in turn can represent anything) and should not be confused with the idea of organizational vision or goals as used in business contexts.

Organizational performance is a value function on the environment (current world), agents and organizational capability, and on the task (desired state of affairs), defined as $\theta\left(w, G_{\leq}, \varphi\right)$, which returns the value of the performance in world $w$ of structured group $G_{\leq}$for $\varphi$. We assume that for each agent and each world, the performance for each atomic proposition $p$ is fixed. Moreover, if $\neg C_{a} \varphi$ then $\theta(w, a, \varphi)=$ $\infty$. By applying the $\theta$ function defined above, one is able to describe the cost of reorganization. This can be used to decide about when to reorganize. A possible strategy to decide whether to realize a reorganization operation $\sigma$ is if $\theta(w, O, \sigma)+\theta\left(w^{\prime}, O^{\prime}, D_{O}\right) \leq \theta\left(w, O, D_{O}\right)$, where $O^{\prime}$ is the organization in $w^{\prime}$ resulting from the realization of $\sigma$ on organization $O$ in $w$. As an example, consider a painters organization such that: $O=\left(\left(\{a, b, c, d\},\left(a \leq_{O} b, a \leq_{O}\right.\right.\right.$ c)) ), $\left.D, S^{0}\right)$, where $D=$ (sand-door $\wedge$ paint-door $)$, and $S^{O}=\left\{R_{a} D, C_{b}\right.$ sand-door, $C_{d}$ paint-door $\}$. It can easily be seen that even though the agents in the organization possess the capabilities to realize the organizational objective, they cannot coordinate efficiently to get it done. Namely, there is no dependency relation from $a$ to $d$, who possesses a necessary capability to get the job done. A possible reorganization is struct $^{+}(O,(a \leq d))$. Furthermore, in other to enable endogenous reorganization, $a$ should have the the capability to do the structuring operation, i.e. $C_{a}$ struct ${ }^{+}(O,(a \leq d))$.

\section{Simulation Framework}

We have developed a simulation framework, Organization Ontology Simulator (OOS) [7], based on the concepts mentioned in section 2. The aim of the OOS is to be able to simulate reorganization in a quick and structured fashion. The framework provides an organizational ontology implementing classes for environment, agent, and structure and related concepts in RDF/RDFS standard format. Simulations in OOS are generated as instances of the organization ontology. Specific organizations are represented as instances of this ontology. OOS converts the ontology instances into a custom JAVA based format, which can be used by the simulation engine. At the moment, we use a Repast $^{1}$ environment to visualize and run the simulations.

Furthermore, OOS provides a custom made plug-in for the 3APL agent platform ${ }^{2}$, which allows the user to create simulations of organization that use software agents with

\footnotetext{
${ }^{1}$ http://repast.sourceforge.net/

${ }^{2}$ http://www.cs.uu.nl/3apl/
}

cognitive capabilities in an open environment. The use of cognitive agents enables the analysis of goal-directed behavior and grounds of this behavior in BDI-logic. Reorganization activities of the staffing- and (part of) the strategy type can be implemented online, because the 3APL platform supplies an open environment. Agents can be taken out and re-inserted in the system, while the simulation is running.

In the following, we illustrate the use of the simulation framework to analyze organizational adaptation. In order to test congruence of organization and environment, our approach was to design two sufficiently different environments, and two organizations that exploit the differences between environments. Using the example organization introduced in section 2 we define the following types of environments and organizations:

- Environments: Generate tasks to be taken up by the organization. Tasks are of 3 types: paint-door, paperwall, tile-floor and we set the frequency at $25 \%$. Two types of environment are considered: heterogenous (Het): all tasks types occur with the same probability, and homogenous (Hom): mostly one type of task: tasks of type paint-door occur in $80 \%$ of the cases; other tasks with $10 \%$ probability each.

- Organizations: have a hierarchical structure and consist of one manager agent and 12 worker agents. Types of organizations are: specialists (Spec): each worker can only perform tasks of one type (4 workers for each type) (time to complete a task is calculated randomly as $T_{s} \in[50,100]$ ), and, generalists (Gen): all workers can perform all tasks, however take longer to complete a task: $T_{g}=2 * T_{s}-25$.

The idea is that a manager receives tasks to be executed and distributes those to a worker with capability to perform that task and is currently free. If no capable agent is free, the manager keeps tasks in a waiting list. There is a penalty for keeping tasks in the waiting list, resulting in less profit. Every completed task gives a profit of $x$ to the organization. For each waiting task the organization looses $x / 10$ each time the task is waiting. In total, four different scenarios are obtained, as follows: Het-Gen, Het-Spec, HomGen, Hom-Spec. We used OOS, described in section 4 to generate (Repast) simulations of the 4 scenarios. Each scenario, with a length of 400 time units, was run 10 times, resulting in 40 different simulations. For each scenario, we calculated the number of completed tasks, the number of waiting tasks, and the average completion length. Figure 1 shows the results obtained for the average of completed tasks. As it can be expected, the performance of Generalist organizations is not influenced by the type of environment. Specialist organizations do not perform well in Homogeneous environments (workers capable of paint-door 


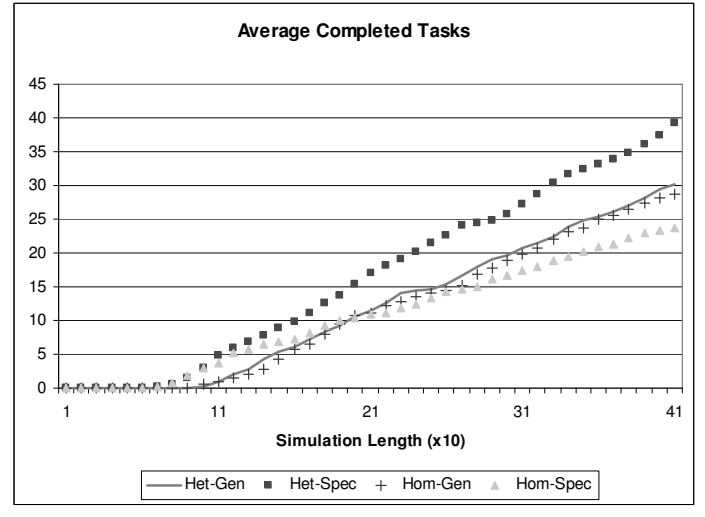

Figure 1. Organizational performance.

are too busy, other workers mostly idle). In such case, reorganization should be considered. We provided manager agents with the capability to check organizational performance and adapt accordingly. In the experiment, manager agents of Specialist organizations on a Homogeneous environment monitor the number of waiting tasks and perform and perform a reorganization action when that number reaches a given threshold. Specifically, when the manager notices that there are 10 or more waiting tasks, it will train one, if any, idle agent to perform the task paint-door. Formally, this is represented as: $E_{m}$ staff $f^{-}(O, a)$ for some agent $a$, followed by $E_{m} \operatorname{staff} f^{+}(O, p)$ where agent $p$ has capabilities $C_{p}$ paint-door. Cost reorganization, that is, the cost of training one agent is $x * 3^{7}$, where $x$ is the task profit. Figure 2 shows the difference of performance between organization Hom-Spec and organization Hom-Spec-Reorg endowed with such reorganization capabilities, for task completion times $T_{s} \in[25,50]$ and task frequency $25 \%$. As

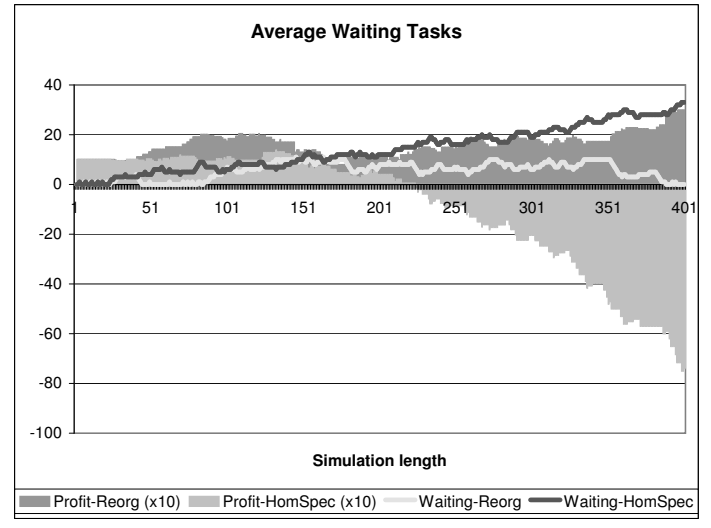

Figure 2. Reorganization effect

it can be seen, reorganization results in less waiting tasks and therefore higher profit due to less penalties. Depending on the values for profit, penalties and reorganization costs, simulations can be used to determine an effective threshold.

\section{Conclusions}

Dynamic reorganization of agent systems is needed in order to enable systems to enforce or adapt to changes in the environment. This issue has been discussed by many researchers and several domain-oriented solutions have been proposed. However, such solutions often lack a formal basis. This makes difficult the development of theories about reorganization and prevents the comparison or adaptation of models to other domains or situations. In this paper we presented a first attempt at a formal model for organizational concepts and the reorganization process, based on modal temporal logic. We further introduced a framework for the rapid development of organizational simulations. This framework provides a structured efficient way to deploy many different organizational and environment designs, by using ontologies describing organization structures, environment characteristics and agent capabilities, and provides semi-automatic means to generate simulations from the ontology instances. Moreover, the simulation framework incorporates the cognitive characteristics of agents. We are currently finalizing the development of the framework and in the near future will be using it for the modelling and analysis of realistic organizations.

Acknowledgements. The authors are grateful to Daghan Acay for his contribution to the development of OOS. This research is funded by the Netherlands Organization for Scientific Research (NWO), through Veni-grant 639.021.509.

\section{References}

[1] K. Carley and D. Svoboda. Modeling organizational adaptation as a simulated annealing process. Sociological Methods \& Research, 25(1):138-168, 1996.

[2] V. Dignum and F. Dignum. Logic for agent organization. In Proc.FAMAS@Durham'007, 2007.

[3] V. Dignum, F. Dignum, and L. Sonenberg. Exploring congruence between organizational structure and task performance: A simulation approach. In O. B. et al., editor, Coordination, Organization, Institutions and Norms in Agent Systems I, Proc. ANIREM'05/OOOP'05, volume 3913 of LNAI, pages 213-230. Springer, 2006.

[4] D. Grossi, F. Dignum, V. Dignum, M. Dastani, and L. Royakkers. Structural aspects of the evaluation of agent organizations. In Coordination, Organization, Institutions and Norms in Agent Systems (COIN II), volume 4386 of LNAI. Springer, 2007.

[5] R. Harrison, Z. Lin, G. Carroll, and K. Carley. Simulation modeling in organizational and management research. Academy of Management Review, to appear, 2007. 
[6] Y. So and E. Durfee. Designing organizations for computational agents. In K. Carley, M. Pritula, and L. Gasser, editors, Simulating Organizations, pages 47-64, 1998.

[7] C. Tick. Organization structure analysis by simulation. Master's thesis, Utrecht University, 2007. 